\title{
Substrate cycling between de novo lipogenesis and lipid oxidation: a thermogenic mechanism against skeletal muscle lipotoxicity and glucolipotoxicity
}

\author{
AG Dulloo $^{1 *}, \mathrm{M} \mathrm{Gubler}^{2}, \mathrm{JP}_{\text {Montani }}{ }^{1}, \mathrm{~J} \mathrm{Seydoux}^{1}$ and G Solinas ${ }^{1}$ \\ ${ }^{1}$ Department of Medicine, Division of Physiology, University of Fribourg, Switzerland; and ${ }^{2}$ Department of Vascular and \\ Metabolic Diseases, Hoffmann-La Roche, Switzerland
}

Life is a combustion, but how the major fuel substrates that sustain human life compete and interact with each other for combustion has been at the epicenter of research into the pathogenesis of insulin resistance ever since Randle proposed a 'glucose-fatty acid cycle' in 1963. Since then, several features of a mutual interaction that is characterized by both reciprocality and dependency between glucose and lipid metabolism have been unravelled, namely:

(i) the inhibitory effects of elevated concentrations of fatty acids on glucose oxidation (via inactivation of mitochondrial pyruvate dehydrogenase or via desensitization of insulin-mediated glucose transport),

(ii) the inhibitory effects of elevated concentrations of glucose on fatty acid oxidation (via malonyl-CoA regulation of fatty acid entry into the mitochondria), and more recently

(iii) the stimulatory effects of elevated concentrations of glucose on de novo lipogenesis, that is, synthesis of lipids from glucose (via SREBP1c regulation of glycolytic and lipogenic enzymes).

This paper first revisits the physiological significance of these mutual interactions between glucose and lipids in skeletal muscle pertaining to both blood glucose and intramyocellular lipid homeostasis. It then concentrates upon emerging evidence, from calorimetric studies investigating the direct effect of leptin on thermogenesis in intact skeletal muscle, of yet another feature of the mutual interaction between glucose and lipid oxidation: that of substrate cycling between de novo lipogenesis and lipid oxidation. It is proposed that this energy-dissipating substrate cycling that links glucose and lipid metabolism to thermogenesis could function as a 'fine-tuning' mechanism that regulates intramyocellular lipid homeostasis, and hence contributes to the protection of skeletal muscle against lipotoxicity.

doi:10.1038/sj.ijo.0802861

Keywords: leptin; AMP-activated protein kinase; PI3-kinase; gluco-lipotoxicity; thermogenesis

\section{Introduction}

Obesity, type 2 diabetes and essential hypertension-three major contributors to cardiovascular diseases - share many epidemiological features. They have long been recognized to be diseases of civilization with a very gradual onset, the result of complex interplay between genetic susceptibility and environmental factors, and with insulin resistance often appearing as a relatively early development in all three entities. $^{1-3}$ What triggers the onset of insulin resistance (ie,

\footnotetext{
*Correspondence: Dr AG Dulloo, Division of Physiology, Department of Medicine, University of Fribourg, Chemin du Musée 5, CH-1700 Fribourg, Switzerland.

E-mail: abdul.dulloo@unifr.ch

Proceedings of the Second Fribourg Obesity Research Conference (FORC2003)
}

the decreased ability of peripheral target tissues to respond properly to normal circulating concentrations of insulin) is not clear. However, since during insulin stimulation the skeletal muscle is the major site for glucose disposal, ${ }^{4}$ defects in insulin-mediated glucose uptake and in its metabolic fate in this tissue are thought to be early events in the pathogenesis of insulin resistance. ${ }^{5,6}$ Indeed, muscle impairments in insulin-mediated glucose transport, glycogen synthesis and glucose oxidative pathways are the central features of individuals at high risk for type 2 diabetes, notably in the lean normoglycemic offspring of diabetic patients and in obese patients. ${ }^{7,8}$ As these population groups also present elevated plasma concentrations of fatty acids and amino acids, ${ }^{9-11}$ these substrates or their metabolites-by interfering with the uptake and metabolism of glucose in skeletal muscle - are thought to play a central role 
in the onset of insulin resistance. ${ }^{12}$ Furthermore, there is also evidence that as chronic hyperglycemia develops, the oversupply of glucose could also, by inhibiting fatty acid oxidation, lead to the accumulation of intramyocellular lipid metabolites that further inhibit glucose uptake/metabolism and hence exacerbate the state of insulin resistance. ${ }^{13-15}$

Since Randle's proposal in 1963 of a glucose-fatty acid cycle that embodies direct competition between substrates for mitochondrial oxidation, a plethora of mechanisms have been put forward to explain how fuel substrates could interfere with glucose disposal in skeletal muscle. ${ }^{12,15}$ Particularly striking is that oversupply of each of the three main fuel substrates can - as depicted in Figure 1-converge towards the accumulation of triglycerides. This can occur not only when the supply of circulating free fatty acid (FFA) is in excess of its oxidation, but also when the supply of amino acids or glucose exceeds the oxidation of these fuels, thereby resulting in the formation of malonyl-CoA which, by inhibiting carnitine palmitoyltransferase-1 (CPT-1), reduces the entry of long-chain fatty acyl-CoAs (FA-CoA) into mitochondrial $\beta$-oxidation. The consequences of such lipid overloading are the provision of lipid substrates (notably FACoA, the product of triglyceride hydrolysis), which, if unoxidized, could underscore the strong association between an elevated intramuscular lipid content and a variety of 'lipotoxic' effects, namely:

(a) impairments in insulin signaling, in particular via inhibition of phosphatidylinositol 3-kinase (PI3K) signaling, a required step in insulin-stimulated glucose transport into muscle, and/or

(b) ceramide synthesis and lipid peroxidation via nonoxidative metabolic pathways, leading to cell dysfunction and death through apoptosis.

Thus, the term 'lipotoxicity' encompasses toxicity that may result not only from lipid overloading induced by excess delivery $v s$ oxidation of circulating FFA, but also from that induced by glucose overloading (gluco-lipotoxicity) and protein overloading (or proteo-lipotoxicity).

While these models of lipotoxicity explain how nutrient supply exceeding oxidation may lead to insulin resistance in skeletal muscle, a fundamental question that arises from a perspective of integrative metabolic regulation is what defense mechanisms may have evolved to enable fuel oxidation to match an increase in fuel supply, and hence contribute to blood glucose homeostasis and protection against muscle lipotoxicity. In addressing this question, we

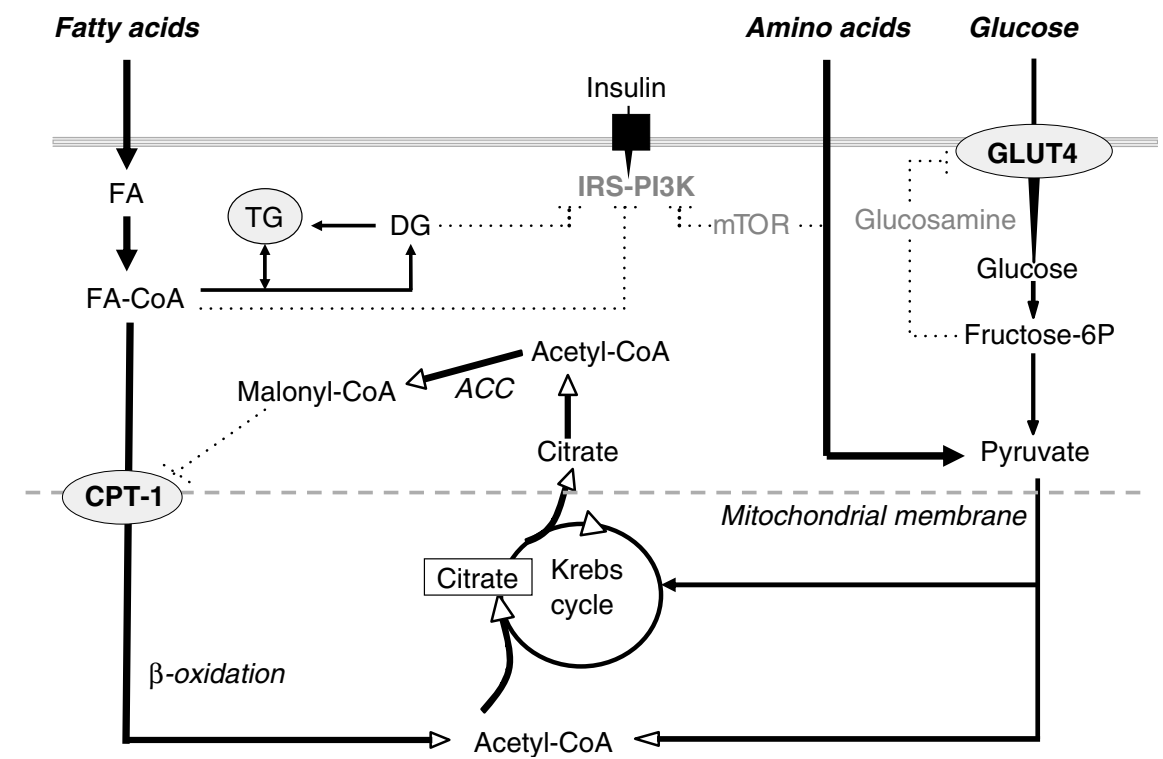

Figure 1 Major pathways by which nutrients oversupply are thought to lead to insulin resistance and lipotoxicity in skeletal muscle: (a) surplus FFA, which is not oxidized through the mitochondrial $\beta$-oxidation pathway, will lead to the enlargement of cytoplasmic pools of triglycerides (TG), diacylglycerols (DG) and longchain FA-CoA. The enlargement of these lipid pools, in particular DG and FA-CoA, could lead to a state of lipotoxicity in two main ways: (i) via activation of various kinases (eg, protein kinase $C$ isoforms, IkB kinase), which in turn lead to inhibition of IRSs associated with PI3K, a required step in insulin-stimulated glucose transport into the muscle cells; and (ii) via ceramide synthesis and lipid peroxidation via nonoxidative metabolic pathways, leading to cell dysfunction and death through apoptosis. (b) An oversupply of amino acids may also impair insulin-mediated glucose uptake by activating the mTOR/p70 S6 protein kinase pathway with consequential inhibition of IRS-PI3K activity, whereas glucose oversupply can lead to inhibition of insulin-stimulated glucose uptake through the formation of glucosamine. (c) Alternatively, surplus glucose or amino acids could also lead to increased conversion of acetyl-CoA to malonyl-CoA, under the catalytic action of ACC. The generation of malonyl-CoA could, by inhibiting CPT-1, reduce mitochondrial fatty acid oxidation with consequential enlargement of the cytoplasmic pool of FA-CoA and DG, thereby creating a state of gluco-lipotoxicity or proteo-lipotoxicity. (d) Absent from this illustration is the pathway through which malonyl-CoA could also serve as a precursor for fatty acid synthesis in skeletal muscle because of the long-held assumption that de novo lipogenesis is practically absent in myocytes. Emerging evidence, however, suggests that this pathway of de novo lipogenesis may have relevance for intramuscular lipid homeostasis, and this is a major point for discussion later in the text. $F A=$ fatty acids. 
first revisit the interactions between glucose and lipid metabolism in skeletal muscle from a perspective of normal physiology of blood glucose homeostasis, and subsequently integrate novel facets of these interactions into a proposal for a thermogenic mechanism of substrate cycling that could offer protection against skeletal muscle lipotoxicity.

\section{Interactions between glucose and lipids: from Randle's viewpoint}

The notion that substrates compete for respiration has a long history. It probably originates from the time of classic calorimetric studies that were being conducted by Benedict nearly $100 \mathrm{y}$ ago, ${ }^{16,17}$ and gained credence in the $1930 \mathrm{~s}$ following the demonstration by $\mathrm{Krebs}^{18}$ of competition between amino acids and glucose in kidney slices, followed by that of Waters et $a l^{19}$ of competition between 3hydroxybutyrate and glucose in dog heart-lung preparations. But it was not until the early 1960s, following the demonstration that lipid fuels inhibit glucose oxidation in rat diaphragm and in perfused heart ${ }^{20,21}$ that Randle $e a^{22,23}$ proposed a 'glucose-fatty acid cycle' as an important set of mechanism by which carbohydrate and fat metabolism interact. The essential components of this cycle have recently been reiterated by $\operatorname{Randle}^{24}$ as follows:

(i) the relationship between glucose and fatty acid oxidation is reciprocal and not dependent,

(ii) in vivo, the oxidation of lipid fuels (fatty acids and ketone bodies) released into the circulation (eg, in starvation or diabetes) may inhibit the catabolism of glucose in muscle, and

(iii) in vitro, the oxidation of fatty acids released from muscle triacylglycerol or intramyocellular triglycerides also inhibit intracellular glucose metabolism.

In an elegant reappraisal of the glucose-fatty acid cycle from a physiological perspective, Frayn ${ }^{25}$ points out that it is appropriately called a 'cycle' because it describes a series of events that interlink carbohydrate and fat metabolism. The operation of this cycle in normal physiology would be as follows:

(a) An elevated glucose concentration (typically in the postprandial state after a meal) stimulates insulin secretion, which then suppresses FFA release from adipose tissue. This removes competition for substrate utilization in skeletal muscle, so that glucose utilization may be stimulated by insulin, unimpeded by high concentrations of fatty acids.

(b) Conversely, when plasma FFA concentrations are high, usually it is because glucose and insulin concentrations are low (typically in the post-absorptive state), fatty acids then naturally become the major fuel for skeletal muscle.

To quote Frayn: ${ }^{25}$ 'This makes perfect physiological sense in terms of blood glucose homeostasis. The 'coarse' control of the reciprocal utilization of glucose and FFA in the body is brought about through insulin secretion: fine-tuning is provided in skeletal muscle'.

\section{Glucose-fatty acid interactions in skeletal muscle: from reciprocality to dependency}

During the past $40 \mathrm{y}$, since the glucose-fatty acid cycle was first proposed, ${ }^{22}$ several major facets of the complex interrelationship between glucose and lipid metabolism have been unraveled in skeletal muscle. As discussed below, they constitute various sets of mechanisms that underscore not only reciprocal relationships between glucose and lipid metabolism but also relationships that show dependency between these two major oxidative fuel substrates for mammalian metabolism.

\section{Inhibitory effects of lipids on glucose metabolism} The inhibitory effects of elevated concentrations of lipid fuels (fatty acids and ketone bodies) on glucose disposal and glucose oxidation in skeletal muscle have been firmly established by numerous studies conducted both in vivo and in vitro. ${ }^{24,25}$ In particular, it has been demonstrated in humans that under in vivo conditions when fatty acid concentrations are elevated (eg, in response to infusion of lipid emulsions together with heparin) whole body and skeletal muscle glucose utilizations are impaired. ${ }^{26-28}$ The mechanisms put forward to explain how fatty acids limit insulin-stimulated glucose utilization fall into two main categories, both of which were first proposed by Randle following the original studies which formed the basis of the glucose-fatty acid cycle, ${ }^{20-23}$ namely: (a) via fatty acidinduced desensitization of insulin-mediated glucose transport and (b) via inhibitory effects of fatty acid oxidation. According to the Randle hypothesis for the latter mechanism, $^{22,24}$ an increase in lipid oxidation in muscle will decrease glucose oxidation by suppression of the mitochondrial pyruvate dehydrogenase complex, with the consequential reduction of glycolytic flux resulting in an increase in glucose-6-phosphate, inhibition of hexokinase activity, and ultimately leading to decreased glucose uptake. Nonoxidative metabolism (storage) of glucose is also, therefore, predicted to be decreased. A variety of model systems have largely confirmed the validity of this Randle's mechanism, at least in terms of the acute effects of fatty acids on muscle glucose metabolism and storage. ${ }^{24,29}$

Since the mid-1990s, however, the mechanisms by which fatty acids may desensitize insulin-mediated glucose transport have received much more attention. According to models that have substantial in vivo and in vitro experimental support, fatty acids or more specifically their metabolites (fatty acyl CoAs, diacyglycerols or even ketone bodies) decrease insulin sensitivity by activation of serine kinase cascades, leading to decreased insulin receptor substrate 
(IRS)-1 tyrosine phosphorylation and decreased IRS-1-associated PI3K activity. ${ }^{6,12,30}$ Other proposed mechanisms have implicated the inhibitory effects of fatty acids (or their metabolites) directly on GLUT4, the major insulin-stimulated glucose transporter. ${ }^{31}$ Taken together, there is still uncertainty regarding which specific metabolites and regulatory pathways are directly responsible for insulin desensitization in response to fatty acids, but the suppressive effects of elevated concentrations of lipids on glucose oxidation in skeletal muscle is unequivocal.

\section{Inhibitory effects of glucose on lipid oxidation}

A turning point in elucidating the reciprocal nature of interactions between glucose and lipid metabolism occurred in the late 1970s following the demonstration of McGarry et $a l^{32,33}$ that high glucose (and insulin) concentrations can suppress hepatic fatty acid oxidation through malonyl-CoA inhibition of CPT-1, a key rate-limiting enzyme that controls the entry of fatty acids into the mitochondrial $\beta$-oxidation system. As this mechanism by which glucose might regulate fatty acid oxidation is an almost exact complement to the mechanism described by Randle and colleagues, it is often referred to as the 'reverse glucose-fatty acid cycle'. MalonylCoA inhibition of fatty acid oxidation may be expected to contribute to the switch to pyruvate oxidation, and conversely, inhibition of acetyl-CoA carboxylase (ACC), which is required to produce malonyl-CoA from acetylCoA, might be expected to facilitate fatty acid oxidation at the expense of glucose oxidation. This reverse glucose-fatty acid cycle can be viewed as further 'fine-tuning' of the balance between glucose and fatty acid metabolism. The question arose nonetheless as how to reconcile the inhibition of mitochondrial fatty acid oxidation by an elevation in malonyl-CoA when this intermediate substrate (that is formed from acetyl-CoA under the catalytic action of ACC) is also an immediate precursor for de novo synthesis of fatty acids. ${ }^{34}$ Such a role for malonyl-CoA both as a precursor of lipid synthesis and an inhibitor of lipid oxidation was subsequently reconciled with the discovery that there are in fact two isoforms of ACC: ${ }^{35,36}$ ACC- 1 expressed predominantly in 'lipogenic' tissues that synthesize large amounts of fatty acids (liver, adipose tissue) and ACC-2 expressed in 'nonlipogenic' tissues such as skeletal muscle and cardiac muscle. More recent advances in the mechanism by which malonyl-CoA is regulated by ACC point to the subcellular localization of the ACC isoforms and to the compartmentalization of malonyl-CoA in the cell. ${ }^{37}$ ACC-1 is thought to reside in the cytoplasm, where it synthesizes the pool of malonyl-CoA that is used for de novo lipogenesis, whereas ACC-2, by contrast, is thought to control the pool of malonyl-CoA that regulates fatty acid oxidation. This isoform-specific role results from its postulated capacity to associate with mitochondria, which juxtaposes the ACC-2 enzyme with mitochondrial CPT- $1 .{ }^{38}$
In skeletal muscle, the suppressive effect of an elevated concentration of glucose on fatty acid oxidation is now recognized to occur via malonyl-CoA-sensitive CPT-1 (malonyl-CoA being synthesized by ACC-2), but the question of whether a cytoplasmic pool of malonyl-CoA might be utilized for fatty acid synthesis has rarely been invokedmost probably because of the long-held assumption that skeletal muscle is not an organ where de novo lipogenesis occurs. Indeed, whenever the expression or activity of a key rate-limiting enzyme for de novo lipogenesis (eg, fatty acid synthase which catalyzes the conversion of malonyl-CoA to fatty acids) was reported in skeletal muscle, this was attributed to artifactual contamination from adipocyte infiltration rather than to de novo lipogenesis occurring in myocytes. ${ }^{39,40}$ Consequently, the importance of skeletal muscle substrate metabolism in the homeostatic control of blood glucose has been viewed entirely from the reciprocal nature of interactions between glucose and lipid metabolism. Recent evidence, however, suggests otherwise. As discussed below, de novo lipogenesis can indeed be shown to occur in muscle cells and to be modulated by factors influencing nutritional status, thereby revealing another facet of interactions between glucose and lipid metabolism that shows dependency between these two fuel substrates in skeletal muscle.

\section{Stimulatory effects of glucose on muscle de novo lipogenesis}

The recent recognition that de novo lipogenesis might have relevance for lipid homeostasis in skeletal muscle stems from the realization that Sterol regulatory element binding protein-1c (SREBP-1c), a member of the family of transcription factors that regulate the expression of genes involved in lipid storage in liver and adipose tissue, is also present in skeletal muscle at a level close to that observed in the liver, ${ }^{41,42}$ and that its dysregulation might lead to increased lipid storage, and hence contribute to the pathogenesis of insulin resistance. There is now evidence both in humans and in rodents that SREBP-1c mediates insulin upregulation of genes encoding glycolytic and lipogenic enzymes in skeletal muscle, ${ }^{42-46}$ but most fascinating are the very recent demonstrations that glucose alone (in the absence of insulin) can stimulate de novo lipogenesis in skeletal muscle cells. The evidence can be summarized as follows:

(i) Using contracting myotubes derived from rat muscle satellite cells, Guillet-Deniau et $a l^{47}$ demonstrated that glucose alone stimulates, more rapidly than insulin, the expression of hexokinase II, fatty acid synthase and ACC-2 proteins, leading to an increased lipogenic flux and intracellular lipid accumulation in these cultured muscle cells. Furthermore, the knockdown of SREBP-1 mRNAs (using an RNA-interference technique) demonstrated that this transcription factor directly mediated the glucose upregulation of lipogenic enzymes in skeletal muscle. 
(ii) In another recent study by Aas et $a l^{48}$ to evaluate the potential effects of hyperglycemia on glucose and lipid metabolism in human muscle cells, it was shown that glucose oversupply leads to increased triglyceride accumulation and the incorporation of glucose into triglycerides, concomitantly with a reduced insulinstimulated glucose uptake and glycogen synthesis, without alterations in fatty acid oxidation. In other words, the increased intramyocellular lipid storage did not result from decreased fatty acid oxidation but from de novo lipogenesis.

Again here, the fact that the formation of malonyl-CoA for de novo synthesis of fatty acids did not result in inhibition of fatty acid oxidation suggests that either (a) the increase in malonyl-CoA may not have exceeded the threshold required for inhibition of CPT-1, or that (b) the malonyl-CoA directed at the synthesis of lipids may have derived essentially from the cytosolic pool synthesized by ACC-1 rather than from the pool localized near to the mitochondria (and synthesized by ACC-2) that are involved in the regulation of fatty acid oxidation. Whatever the explanation, it is clear that de novo lipogenesis, although low in skeletal muscle, can be markedly stimulated in muscle cells, particularly under conditions of high glucose (and/or high insulin) concentrations. These findings in primary muscle cell cultures are consistent with recent data from our laboratory, which demonstrate that de novo lipogenesis can also occur in intact skeletal muscle. As shown in Figure 2, incubation of intact mouse muscle preparations in buffer containing ${ }^{14} \mathrm{C}$-labeled glucose resulted in the production of ${ }^{14} \mathrm{C}$-labeled lipids corresponding to FFAs, diglycerides and triglycerides when analyzed by thin-layer chromatography, and that insulin increased the synthesis of lipids from glucose in the soleus muscle (predominantly oxidative fibre type), and to a lesser extent in the extensor digitorium longus (EDL) muscle (predominantly glycolytic fibre type). This experiment demonstrates that de novo lipogenesis can occur in skeletal muscle independently of muscle type and that it can be stimulated by insulin as in the liver and adipose tissue.

What could then be the normal physiological significance of de novo lipogenesis in skeletal muscle? Extrapolated to conditions of postprandial elevation in blood glucose and insulin (particularly after a high-carbohydrate meal), de novo lipogenesis in skeletal muscle, like in the liver, could also contribute to blood glucose homeostasis by disposing some of the excess circulating glucose as muscle triglycerides, particularly if the glycogen stores are full. In other words, de novo lipogenesis in myocytes may provide another sink for glucose disposal through skeletal muscle. However, given the well-established connection between an elevated intramyocellular lipid and insulin resistance, the concept of an increase in de novo lipogenesis in skeletal muscle as an 'adaptive' physiological reaction to post-prandial hyperglycemia is only tenable if the lipids synthesized de novo were to be subsequently oxidized-that is, through an increase in

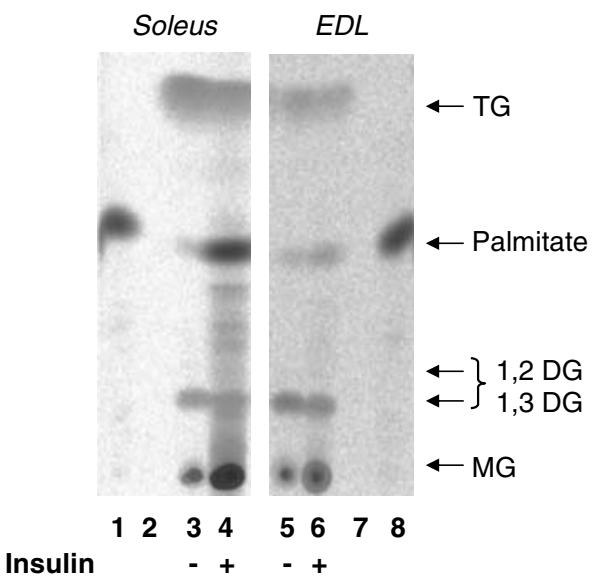

Figure 2 De novo lipogenesis in intact skeletal muscle. Phosphor imaging of thin layer chromatography showing conversion of $D$ - $\left[{ }^{14} \mathrm{C}\right]$-glucose into $\left[{ }^{14} \mathrm{C}\right]$ lipids in mouse soleus and EDL, predominantly oxidative and glycolytic muscles, respectively, incubated in Krebs-Ringer buffer containing $5 \mathrm{mM}$ glucose supplemented with D-[ $\left[{ }^{14} \mathrm{C}\right]$-glucose: lanes 3 and 5 contain lipids extracted from muscles incubated in control buffer, and lanes 4 and 6 contain lipids extracted from muscles incubated with $100 \mathrm{nM}$ insulin; lipid standards are as follows: lane 1 and $8,{ }^{14} \mathrm{C}$-labeled palmitate; lanes 2 and 7 , mixture of unlabeled mono- (MG), di- (DG) and tri-glycerides (TG) that were detected colorimetrically. For details of incubation and analysis, see Solinas et al. ${ }^{63}$

muscle thermogenesis. Two separate lines of investigations converge to support this contention. First, a link between glucose disposal and a disproportionate increase in skeletal muscle thermogenesis (unaccounted for by the energy costs for glucose uptake and storage as glycogen) has indeed been demonstrated in humans during measurements of forearm $\mathrm{O}_{2}$ consumption for several hours after an oral glucose load. ${ }^{49}$ Second, as discussed below, recent work from our laboratory investigating the mechanisms by which leptin- another hormone whose blood concentrations rise during the post-prandial state $\mathrm{s}^{50,51}$ - may interact with insulin to stimulate thermogenesis in skeletal muscle, suggests the possible existence of a thermogenic pathway of substrate cycling in which lipids derived from glucose (ie, by de novo lipogenesis) are subsequently oxidized.

\section{Substrate cycling between de novo lipogenesis and lipid oxidation}

It is now well established that the adipocyte-derived hormone leptin, which is well known for its central role in body weight regulation in part via its control over thermogenesis, ${ }^{52-55}$ also plays an important role in blood glucose homeostasis and in the protection of insulin-sensitive tissues against excessive ectopic lipid storage by regulating the partitioning of fatty acid away from storage towards oxidation. ${ }^{56-58}$ As depicted in Figure 3, the earlier demonstrations that leptin can act directly on skeletal muscle, specifically via the long form of the leptin receptor (ObRb), to stimulate glucose utilization in a PI3K-dependent manner ${ }^{59,60}$ and lipid oxidation via the activation of AMP-activated protein 


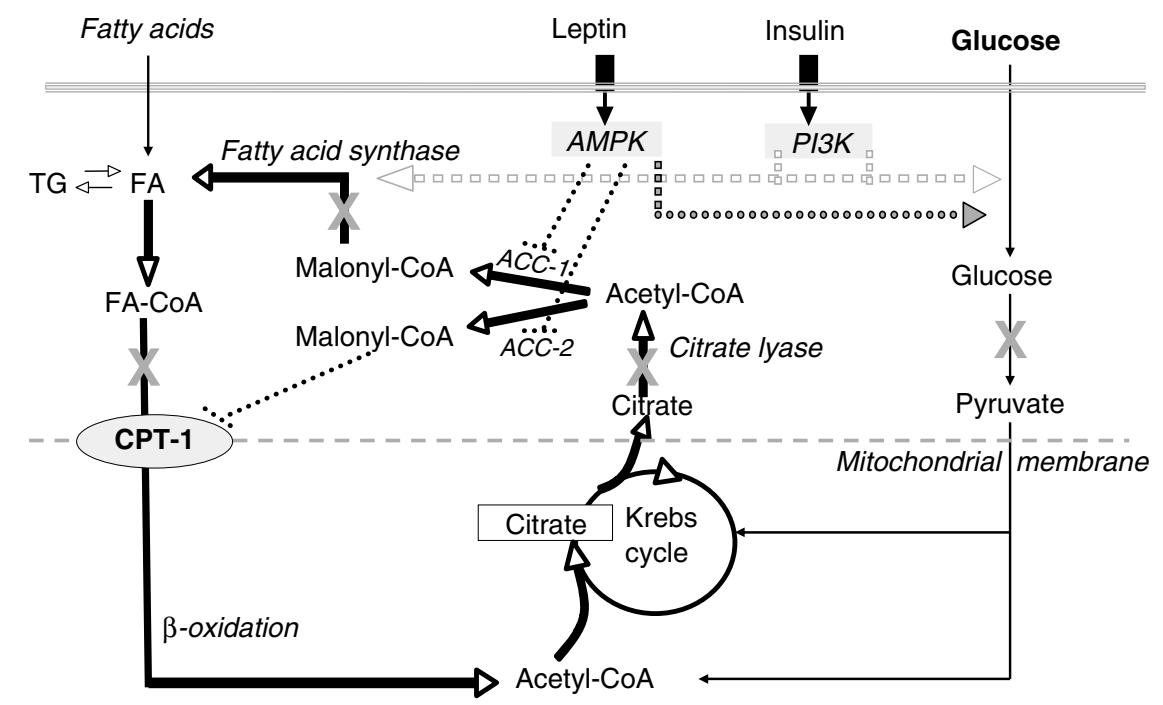

Figure 3 Biochemical model integrating an energy-dissipating substrate cyling between de novo lipogenesis and lipid oxidation (orchestrated by PI3K and AMPK signaling) in skeletal muscle. The possible existence of this substrate cycling rests upon data from calorimetric studies showing that the direct effect of leptin on skeletal muscle thermogenesis is potentiated by insulin and inhibited by pharmacological interference at any one of the key control points in the flux of substrates denoted by symbol (X), namely: (i) with glucose metabolism using 2-deoxyglucose, (ii) with the conversion of citrate to acetyl-CoA using hydroxy-citrate, an inhibitor of citrate lyase, (iii) with the conversion of malonyl-CoA to fatty acids using cerulenin, an inhibitor of fatty acid synthase and (iv) with the entry of fatty acid into mitochondrial $\beta$-oxidation pathway using etomoxir, an inhibitor of CPT-1. It is postulated that other hormones (eg, catecholamines, adiponectin), which have been shown to activate AMPK signaling in skeletal muscle, could also interact with insulin to influence the rate of this thermogenic substrate cycling, and hence play a role in intramuscular lipid homeostasis. See text for details. FA = fatty acids; TG = triglycerides.

kinase (AMPK) leading to inhibition of $\mathrm{ACC} \mathrm{C}^{58,61}$ prompted us to investigate whether leptin could also exert direct control on thermogenesis in skeletal muscle. Using a method that involves repeated measurements of $\mathrm{O}_{2}$ consumption in intact muscle perifused ex vivo with Krebs-Ringer buffer, we found that leptin could directly stimulate thermogenesis in skeletal muscle via ObRb, ${ }^{62}$ and that this thermogenic effect of leptin, which requires PI3K activity (since it is inhibited by wortmannin), is potentiated by insulin, a potent activator of PI3K. A requirement for AMPK activation and for entry of fatty acids through the mitochondrial $\beta$-oxidation pathway was also suggested by the fact that leptin-induced thermogenesis was inhibited either in the presence of araA (an inhibitor of AMPK) or by addition of etomoxir, an inhibitor of CPT- $1 .{ }^{63}$ In other experiments that involved interference with key control points of intermediary metabolism in order to investigate the nature of an 'apparent' link between leptin's direct effects on skeletal muscle to stimulate glucose metabolism, ${ }^{58-60}$ lipid oxidation ${ }^{57-61}$ and thermogenesis, ${ }^{62}$ evidence emerged of a requirement for de novo lipogenesis in the mechanism by which leptin stimulates muscle thermogenesis. ${ }^{63}$ Specifically, the direct thermogenic effect of leptin on skeletal muscle $\mathrm{O}_{2}$ consumption was found to be completely inhibited by the addition of any one of the following compounds that would inhibit the synthesis of lipids from glucose, namely (a) 2-deoxyglucose, which interferes with glucose metabolism, (b) hydroxy-citrate, which inhibits citrate lyase and hence the conversion of citrate to acetyl-CoA and (c) cerulenin, which inhibits fatty acid synthase and hence the conversion of malonyl-CoA to fatty acids. Taken together, these studies suggest that the direct effect of leptin in stimulating thermogenesis in skeletal muscle could be mediated by substrate cycling between de novo lipogenesis and lipid oxidation, and that the orchestration of this substrate cycling requires both PI3K and AMPK signaling.

It is proposed that in this substrate cycle (depicted in Figure 3), which underscores the interdependency between glucose, lipids and thermogenesis, acetyl-CoA produced from glucose and fatty acid oxidation will overload the Krebs cycle. This will result in excess mitochondrial citrate, which, in the cytoplasm, will exert an allosteric activation of ACCs and, at the same time, under the action of citrate lyase, will provide acetyl-CoA to ACCs for the synthesis of malonyl-CoA. The latter will serve as the main substrate for fatty acid synthase, thereby producing a new pool of fatty acids. Glucose plays a central role in this cycle as a source of acetyl-CoA, Krebs cycle intermediates and NADPH molecules, which are required for the synthesis of FAs. It might also function as a stimulator of de novo lipogenesis, based upon the above-mentioned recent demonstrations in muscle satellite cells ${ }^{47,48}$ that glucose, even in the absence of insulin, stimulates the gene expression of SREBP-1c as well as key genes encoding glycolytic and lipogenic enzymes, leading to an increased lipogenic flux.

It is postulated that PI3K and AMPK signaling could orchestrate this futile cycle between de novo fatty acid synthesis and fatty acid oxidation. On the one hand, 
activation of AMPK, by phosphorylating ACCs, will counterbalance the stimulatory action of citrate on ACCs to result in reduced malonyl-CoA concentration, disinhibition of CPT-1 and increased fatty acid oxidation, which in turn will lead to the production of acetyl-CoA and consequently overloading of the Krebs cycle. On the other hand, PI3K activity (basal or insulin-stimulated) will increase glucose entry and allow the excess citrate to enter pathways leading to the synthesis of fatty acids. This would occur despite AMPK-induced reduction in malonyl-CoA since it is known that full phosphorylation of ACCs by AMPK results in an inhibition of ACC activities only by $50-60 \%{ }^{64,65}$ Such partial inhibition of ACCs is expected to redirect the flux of acetyl-CoA and malonyl-CoA towards fatty acid oxidation, but would still allow substantial rate of fatty acid synthesis, particularly in the presence of high levels of citrate. Alternatively, the insulin-leptin interactions in skeletal muscle may orchestrate the regulation of distinct intracellular pools of malonylCoA via maintenance of the ACC-1-generated 'lipogenic' pool (through PI3 kinase signaling) and diminution of the ACC-2-generated 'CPT-1-sensitive' pool (via AMPK signaling). One can also entertain the interesting possibility that, in skeletal muscle, this substrate cycling is also activated in response to other hormones and neurotransmitters (eg, adiponectin, catecholamines) particularly since adiponectin, as well as adrenergic agonists, can also stimulate AMPK activity, glucose utilization and fatty acid oxidation in skeletal muscle or adipose tissue. ${ }^{61,66-69}$ This substrate cycling between de novo lipogenesis and lipid oxidation could therefore constitute a thermogenic effector in skeletal muscle. Theoretically, the synthesis of one molecule of palmitic acid from acetyl-CoA and its re-oxidation to acetylCoA would cost at least 14 molecules of ATP. Repeated recycling of acetyl-CoA through the flux of substrates across lipogenesis, followed by lipid oxidation, could therefore constitute an important thermogenic pathway that provides a sink not only for intramuscular glucose disposal, but also for dissipating excess lipids.

The existence of this futile cycle in skeletal muscle provides a mechanistic link between glucose utilization, fatty acid metabolism and thermogenesis, analogous to the relation between substrate metabolism and thermogenesis in brown adipose tissue. In this tissue, whose primary function is to produce heat for thermoregulation in human newborns and small mammals, it has long been known that much of the fuel for thermogenesis also derives from glucose being first converted to lipids before being oxidized. ${ }^{70-73}$ More direct evidence that de novo lipogenesis is in fact critical for brown adipose tissue thermogenesis can be derived from recent studies in cold-exposed mice indicating that pharmacological suppression of de novo lipogenesis leads to marked reductions in brown adipose tissue thermal response to noradrenaline infusion (despite normal UCP1 levels) and resulted in the development of hypothermia. ${ }^{74}$ One can therefore also refer to thermogenesis in brown adipose tissue as dependent upon substrate cycling between de novo lipogenesis and lipid oxidation, but whose rate is greatly amplified under sympathetic activation of mitochondrial proton leak in this tissue.

\section{Interdependency between glucose, lipids and thermogenesis}

Thus, although research towards understanding how the major oxidative fuels interact for combustion in skeletal muscle has been dominated by concepts of reciprocal interactions between glucose and lipid oxidation, the notion of interdependency between glucose uptake/metabolism, lipid oxidation and thermogenesis is not entirely new. It has been discussed by Masaro in the early 1960s in the context of a role for adipose tissue metabolism in nonshivering thermogenesis pertaining to body temperature regulation. ${ }^{70}$ Furthermore, this 'dependency' interaction between these two substrates and thermogenesis is well recognized at the whole-body level, and is attributed to activation of a neuroendocrine network (comprising insulin, leptin and the sympathoadrenal system), which plays a pivotal role in several overlapping regulatory systems: that of blood glucose, body temperature, body weight and more recently intramyocellular lipids. ${ }^{56-58}$ Interference with any of these hormones or their actions leads to major impairments in all these overlapping regulatory functions. In particular, the absence of functional leptin (eg, in the $o b / o b$ mouse) or dysfunctional leptin signaling (eg, in $d b / d b$ mice or $f a / f a$ rats) leads not only to impairments in thermogenesis that contribute to obesity, but also to hyperglycemia and excessive accumulation of lipids in nonadipose tissues, including the skeletal muscle. ${ }^{56-58}$ The fact that, in these animal models, the stimulation of thermogenesis induced by leptin replacement ${ }^{58}$ or by treatment with $\beta 3$ adrenoceptor agonists ${ }^{75}$ improves insulin sensitivity and reduces ectopic fat storage at low-dose levels or over time periods that do not affect body weight strongly suggests that impaired thermogenesis might be an early event in the dysregulation of blood glucose and intramyocellular lipids. To date, however, the molecular mechanisms underlying thermogenesis in tissues other than the brown adipose tissue remains elusive, amid continuing controversies concerning the physiological roles of UCP2 and UCP3 (homologs of UCP1) as effectors of skeletal muscle thermogenesis. $^{76,77}$ The energy-dissipating substrate cycle that links glucose and lipid metabolism to thermogenesis in skeletal muscle (depicted in Figure 3) provides a novel molecular mechanism of thermogenesis through which this abovementioned neuroendocrine network-operating through insulin, leptin and catecholamines-overlaps in the regulation of body weight, blood glucose and intramyocellular lipids, and hence in the protection against obesity, hyperglycemia and lipotoxicity. Perturbations in this neuroendocrine network (and intracellular signaling system) that exerts control over this substrate cycling may thus be early events that lead to intramyocellular lipid accumulation, and 
hence to the consequential effects of this state of lipotoxicity on the onset and exacerbation of insulin resistance.

\section{Acknowledgements}

This work was supported by the Swiss National Science Foundation (grant \# 3200B0-102156).

\section{References}

1 Neel JV, Weder AB, Julius S. Type II diabetes, essential hypertension, and obesity as 'syndromes of impaired genetic homeostasis': the 'thrifty genotype' hypothesis enters the 21st century. Perspect Biol Med 1998; 42: 44-75.

2 Landsberg L. Diet, obesity and hypertension: a hypothesis involving insulin, the sympathetic nervous system and adaptive thermogenesis. Q J Med 1986; 61: 1081-1090.

3 Landsberg L. Insulin-mediated sympathetic stimulation: role in the pathogenesis of obesity-related hypertension (or, how insulin affects blood pressure, and why). J Hypertens 2001; 19: 523-528.

4 DeFronzo RA, Jacot E, Jequier E, Maeder E, Wahren J, Felber JP. The effect of insulin on the disposal of intravenous glucose. Results from indirect calorimetry and hepatic and femoral venous catheterization. Diabetes 1981; 30: 1000-1007.

5 DeFronzo RA. Lilly lecture 1987. The triumvirate: beta-cell, muscle, liver. A collusion responsible for NIDDM. Diabetes 1988; 37: 667-687.

6 Petersen KF, Schulman GI. Pathogenesis of skeletal muscle insulin resistance in type 2 diabetes mellitus. Am J Cardiol 2002; 90 (Suppl): 11G-18G.

7 Rothman DL, Magnusson I, Cline G, Gerard D, Kahn CR, Shulman RG, Shulman GI. Decreased muscle glucose transport/ phosphorylation is an early defect in the pathogenesis of noninsulin-dependent diabetes mellitus. Proc Natl Acad Sci USA 1995; 92: 983-987.

8 Roden M, Shulman GI. Applications of NMR spectroscopy to study muscle glycogen metabolism in man. Annu Rev Med 1999; 50: 277-290.

9 Reaven GM. Banting lecture 1988. Role of insulin resistance in human disease. Diabetes 1988; 37: 1595-1607.

10 Felig P, Marliss E, Cahill Jr GF. GF Plasma amino acid levels and insulin secretion in obesity. N Engl J Med 1969; 281: 811-816.

11 Felber JP, Golay A. Pathways from obesity to diabetes. Int J Obes Relat Metab Disord 2002; 26 (Suppl 2): S39-S45.

12 Krebs M, Roden M. Nutrient-induced insulin resistance in human skeletal muscle. Curr Med Chem 2004; 11: 907-908.

13 Richter EA, Hansen BF, Hansen SA. Glucose-induced insulin resistance of skeletal-muscle glucose transport and uptake. Biochem J 1988; 252: 733-737.

14 Laybutt DR, Schmitz-Peiffer C, Saha AK, Ruderman NB, Biden TJ, Kraegen EW. Muscle lipid accumulation and protein kinase C activation in the insulin-resistant chronically glucose-infused rat. Am J Physiol 1999; 277: E1070-E1076.

15 Hegarty BD, Furler SM, Ye J, Cooney GJ, Kraegen EW. The role of intramuscular lipid in insulin resistance. Acta Physiol Scand 2003; 178: 373-383.

16 Benedict FG, Joslin EP. Metabolism in Diabetes Mellitus. Carnegie Institute of Washington: Washington DC, USA, Publication 136; 1910. pp 3-234.

17 Cahill Jr GF, Owen OE. Some observations on carbohydrate metabolism in man. In: Dickens F, Randle PJ, Whelan WJ (eds). Carbohydrate metabolism and its disorders. Academic Press: London; 1968. pp 497-522.

18 Krebs HA. Metabolism of amino acids. III. Deamination of amino acids. Biochem J 1935; 29: 1620-1644.
19 Waters ET, Fletcher JP, Mirsky IA. Relation between carbohydrate and 3-hydroxybutyrate utilization by heart lung preparations. Am J Physiol 1938; 122: 542-546.

20 Newsholme EA, Manchester KL, Randle PJ. Inhibition of the phosphofructokinase reaction in perfused rat heart by respiration of ketone bodies, fatty acids and pyruvate. Nature 1962; 193: 270-271.

21 Garland PB, Newsholme EA, Randle PJ. Effect of fatty acids, ketone bodies, diabetes and starvation on pyruvate metabolism in rat heart and diaphragm muscle. Nature 1962; 195: 381-383.

22 Randle PJ, Garland PB, Hales CN, Newsholme EA. The glucosefatty acid cycle: its role in insulin sensitivity and metabolic disturbances of diabetes mellitus. Lancet 1963; i: 785-789.

23 Randle PJ, Garland PB, Hales CN, Newsholme EA, Denton RM, Pogson CI. Interactions of metabolism and physiological role of insulin. Recent Prog Horm Res 1966; 22: 1-48.

24 Randle PJ. Regulatory interactions between lipids and carbohydrates: the glucose fatty acid cycle after 35 years. Diabetes Metab Rev 1998; 14: 263-283.

25 Frayn KN. The glucose-fatty acid cycle: a physiological perspective. Biochem Soc Trans 2003; 31: 1115-1119.

26 Ferrannini E, Barrett EJ, Bevilacqua S, DeFronzo RA. Effect of fatty acids on glucose production and utilization in man. J Clin Invest 1983; 72: 1737-1747.

27 Kelley DE, Mokan M, Simoneau JA, Mandarino LJ. Interaction between glucose and free fatty acid metabolism in human skeletal muscle. J Clin Invest 1993; 92: 91-98.

28 Boden G, Chen X, Ruiz J, White JV, Rossetti L. Mechanisms of fatty acid-induced inhibition of glucose uptake. J Clin Invest 1994; 93: 2438-2446.

29 Boden G. Interaction between free fatty acids and glucose metabolism. Curr Opin Clin Nutr Metab Care 2002; 5: 545-549.

30 Bjornholm M, Kawano Y, Lehtihet M, Zierath JR. Insulin receptor substrate-1 phosphorylation and phosphatidylinositol 3-kinase activity in skeletal muscle from NIDDM subjects after in vivo insulin stimulation. Diabetes 1997; 46: 524-527.

31 Kahn BB. Glucose transport: pivotal step in insulin action. Diabetes 1996; 45: 1644-1654.

32 McGarry JD, Mannaerts GP, Foster DW. A possible role for malonyl-CoA in the regulation of hepatic fatty acid oxidation and ketogenesis. J Clin Invest 1977; 60: 265-270.

33 McGarry JD. Glucose-fatty acid interactions in health and disease. Am J Clin Nutr 1998; 67 (Suppl): 500S-504S.

34 Nugteren $\mathrm{DH}$. The enzymic chain elongation of fatty acids by ratliver microsomes. Biochim Biophys Acta 1965; 106: 280-290.

35 Bianchi A, Evans JL, Iverson AJ, Nordlund AC, Watts TD, Witters LA. Identification of an isozymic form of acetyl-CoA carboxylase. J Biol Chem 1990; 265: 1502-1509.

36 Ha J, Lee JK, Kim KS, Witters LA, Kim KH. Cloning of human acetyl-CoA carboxylase-beta and its unique features. Proc Natl Acad Sci USA 1996; 93: 11466-11470.

37 Abu-Elheiga L, Brinkley WR, Zhong L, Chirala SS, Woldegiorgis G, Wakil SJ. The subcellular localization of acetyl-CoA carboxylase 2. Proc Natl Acad Sci USA 2000; 97: 1444-1449.

38 Abu-Elheiga L, Matzuk MM, Abo-Hashema KA, Wakil SJ. Continuous fatty acid oxidation and reduced fat storage in mice lacking acetyl-CoA carboxylase 2. Science 2001; 291: 2613-2616.

39 Alam N, Saggerson ED. Malonyl-CoA and the regulation of fatty acid oxidation in soleus muscle. Biochem J 1998; 334: 233-241.

40 Jayakumar A, Tai MH, Huang WY, al-Feel W, Hsu M, Abu-Elheiga L, Chirala SS, Wakil SJ. Human fatty acid synthase: properties and molecular cloning. Proc Natl Acad Sci USA 1995; 92: 8695-8699.

41 Shimomura I, Shimano H, Horton JD, Goldstein JL, Brown MS Differential expression of exons $1 \mathrm{a}$ and $1 \mathrm{c}$ in mRNAs for sterol regulatory element binding protein-1 in human and mouse organs and cultured cells. J Clin Invest 1997; 99: 838-845.

42 Ducluzeau PH, Perretti N, Laville M, Andreelli F, Vega N, Riou JP, Vidal H. Regulation by insulin of gene expression in human 
skeletal muscle and adipose tissue. Evidence for specific defects in type 2 diabetes. Diabetes 2001; 50: 1134-1142.

43 Sewter C, Berger D, Considine RV, Medina G, Rochford J, Ciaraldi T, Henry R, Dohm L, Flier JS, O’Rahilly S, Vidal-Puig AJ. Human obesity and type 2 diabetes are associated with alterations in SREBP1 isoform expression that are reproduced ex vivo by tumor necrosis factor-alpha. Diabetes 2002; 51: 1035-1041.

44 Guillet-Deniau I, Mieulet V, Le Lay S, Achouri Y, Carre D, Girard J, Foufelle F, Ferre P. Sterol regulatory element binding protein-1c expression and action in rat muscles: insulin-like effects on the control of glycolytic and lipogenic enzymes and UCP3 gene expression. Diabetes 2002; 51: 1722-1728.

45 Bizeau ME, MacLean PS, Johnson GC, Wei Y. Skeletal muscle sterol regulatory element binding protein-1c decreases with food deprivation and increases with feeding in rats. J Nutr 2003; 133: 1787-1792.

46 Commerford SR, Peng L, Dube JJ, O'Doherty RM. In vivo regulation of SREBP-1c in skeletal muscle: effects of nutritional status, glucose, insulin, and leptin. Am J Physiol 2004; 287: R218-R227.

47 Guillet-Deniau I, Pichard AL, Koné A, Esnous C, Nieruchalski M, Girard J, Prip-Buus C. Glucose induces de novo lipogenesis in rat muscle satellite cells through a sterol-regulatory-element-binding-protein-1c-dependent pathway. J Cell Sci 2004; 177: 1937-1944

48 Aas V, Kase ET, Solberg R, Jensen J, Rustan AC. Chronic hyperglycaemia promotes lipogenesis and triacylglycerol accumulation in human skeletal muscle cells. Diabetologia 2004; 47: 1452-1461

49 Astrup A, Bulow J, Christensen NJ, Madsen J, Quaade F. Facultative thermogenesis induced by carbohydrate: a skeletal muscle component mediated by epinephrine. Am J Physiol 1986; 250: E226-E229.

50 Schoeller DA, Cella LK, Sinha MK, Caro JF. Entrainment of the diurnal rhythm of plasma leptin to meal timing. J Clin Invest 1997; 100: 1882-1887.

51 Evans K, Clark ML, Frayn KN. Carbohydrate and fat have different effects on plasma leptin concentrations and adipose tissue leptin production. Clin Sci 2001; 100: 493-498.

52 Pelleymounter MA, Cullen MJ, Baker MB, Hecht R, Winters D, Boone T, Collins F. Effects of the obese gene product on body weight regulation in ob/ob mice. Science 1995; 269: 540-543.

53 Halaas JL, Gajiwala KS, Maffei M, Cohen SL, Chait BT, Rabinowitz D, Lallone RL, Burley SK, Friedman JM. Weight-reducing effects of the plasma protein encoded by the obese gene. Science 1995; 269: 543-546.

54 Mistry AM, Swick AG, Romsos DR. Leptin rapidly lowers food intake and elevates metabolic rates in lean and ob/ob mice. J Nutr 1997; 127: 2065-2072.

55 Rosenbaum M, Murphy EM, Heymsfield SB, Matthews DE, Leibel RL. Low dose leptin administration reverses effects of sustained weight-reduction on energy expenditure and circulating concentrations of thyroid hormones. J Clin Endocrinol Metab 2002; 87: 2391-2394.

56 Unger RH, Orci L. Diseases of liporegulation: new perspective on obesity and related disorders. FASEB J 2001; 15: 312-321.

57 Ravussin E, Smith SR. Increased fat intake, impaired fat oxidation, and failure of fat cell proliferation result in ectopic fat storage, insulin resistance, and type 2 diabetes mellitus. Ann N Y Acad Sci 2002; 967: 363-378.

58 Muoio DM, Lynis Dohm G. Peripheral metabolic actions of leptin. Best Pract Res Clin Endocrinol Metab 2002; 16: 653-666.

59 Muoio DM, Dohn GL, Fiedorek FT, Tapscott EB, Coleman RA. Leptin directly alters lipid partitioning in skeletal muscle. Diabetes 1997; 46: 1360-1363.
60 Ceddia RB, William Jr WN, Curi R. The response of skeletal muscle to leptin. Front Biosci 2001; 6: D90-D97.

61 Minokoshi Y, Kim YB, Peroni OD, Fryer LG, Muller C, Carling D, Kahn BB. Leptin stimulates fatty-acid oxidation by activating AMP-activated protein kinase. Nature 2002; 415: 339-343.

62 Dulloo AG, Stock MJ, Solinas G, Boss O, Montani JP, Seydoux J. Leptin directly stimulates thermogenesis in skeletal muscle. FEBS Lett 2002; 515: 109-113.

63 Solinas G, Summermatter S, Mainieri D, Gubler M, Pirola L, Wymann MP, Rusconi S, Montani JP, Seydoux J, Dulloo AG. The direct effect of leptin on skeletal muscle thermogenesis is mediated by substrate cycling between de novo lipogenesis and lipid oxidation. FEBS Lett 2004; 577: 539-544.

64 Winder WW, Hardie DG. Inactivation of acetyl-CoA carboxylase and activation of AMP-activated protein kinase in muscle during exercise. Am J Physiol 1996; 270: E299-E304.

65 Gubler M, Westerberg R, Andjelkovic M, Mizrahi J. Functional characterization of human ACC2 enzyme and elucidation of its role in the regulation of mitochondrial fatty acid oxidation. Keystone Conference on Obesity: New Insights into Pathogenesis and Treatment.Keystone, 2003,Poster Abstract 214.

66 Yamauchi T, Kamon J, Minokoshi Y, Ito Y, Waki H, Uchida S, Yamashita S, Noda M, Kita S, Ueki K, Eto K, Akanuma Y, Froguel P, Foufelle F, Ferre P, Carling D, Kimura S, Nagai R, Kahn BB, Kadowaki T. Adiponectin stimulates glucose utilization and fattyacid utilization by activating AMP-activated protein kinase. Nat Med 2002; 8: 1288-1295.

67 Tomas E, Tsao TS, Saha AK, Murrey HE, Zhang CC, Itani SI, Lodish HF, Ruderman NB. Enhanced muscle fat oxidation and glucose transport by ACRP30 globular domain: acetylcoA carboxylase inhibition and AMP-activated protein kinase activation. Proc Natl Acad Sci USA 2002; 99: 16309-16313.

68 Moule SK, Denton M. The activation of p38 MAPK by the $\beta$ adrenergic agonist isoproterenol in rat epididymal fat cells. FEBS Lett 1998; 439: 287-290.

69 Yin W, Mu J, Birnbaum MJ. Role of AMP-activated protein kinase in cyclic AMP-dependent lipolysis in 3T3-L1 adipocytes. J Biol Chem 2003; 278: 43074-43080.

70 Masaro EJ. Role of lipogenesis in nonshivering thermogenesis. Fed Proc 1963; 22: 868-873.

71 Ma SWY, Foster DO. Uptake of glucose and release of fatty acids and glycerol by rat brown adipose tissue in vivo. Can J Physiol Pharmacol 1985; 64: 609-614.

72 Trayhurn P. Fuel selection in brown adipose tissue. Proc Nutr Soc 1995; 54: 39-47.

$73 \mathrm{Yu}$ XX, Lewin DA, Forrest W, Adams SH. Cold elicits the simultaneous induction of fatty acid synthesis and $\beta$-oxidation in murine brown adipose tissue: prediction from differential gene expression and confirmation in vivo. FASEB J 2002; 16: $155-168$.

74 Christoffolete MA, Linardi CC, de Jesus L, Ebina KN, Carvalho SD, Ribeiro MO, Rabelo R, Curcio C, Martins L, Kimura ET, Bianco AC. Mice with targeted disruption of the Dio2 gene have coldinduced overexpression of the uncoupling protein 1 gene but fail to increase brown adipose tissue lipogenesis and adaptive thermogenesis. Diabetes 2004; 53: 577-584.

75 Arch JRS. $\beta$-3-adrenoceptor agonists: potential, pitfalls and progress. Eur J Pharmacol 2002; 440: 99-107.

76 Samec S, Seydoux J, Dulloo AG. Role of UCP homologues in skeletal muscle and brown adipose tissue: mediators of thermogenesis or regulators of lipids as fuel substrate? FASEB J 1998; 12: $715-724$.

77 Dulloo AG, Seydoux J, Jacquet J. Adaptive thermogenesis and uncoupling proteins: a reappraisal of their roles in fat metabolism and energy balance. Physiol Behav 2004. (in press). 